\title{
Contribution to the Ring-Core Fluxgate Theory
}

\author{
Pavel Ripka
}

Czech Technical University, Faculty of Electrical Eng., Dept. of Measurements, Suchbatarova 2, 16627 Praha 6, Czechoslovakia

Received September 8, 1987; accepted January 20, 1989

P.Ripka: Contribution to the Ring-Core Fluxgate Theory, Physica Scripta, 40 (1989) 544-547

\begin{abstract}
Existing theories for open-core fluxgate are not fitting experimental results in the case of ring-core sensors. Simple description based on transfer functions which takes into account the interaction between two half-cores is presented. Measured inner loops and dynamical transfer functions justified this approach. Flux-evaluating fluxgate was proposed for simple instruments. Its main advantage is its insensitivity to excitation current field parameter variations, but it has a threshold level of about $100 \mathrm{nT}$ in present time. Methods of calibration and noise evaluation of sensors are also mentioned.
\end{abstract}

\section{Introduction}

Ring-core fluxgate has been widely used since the end of the sixties. Although its sensitivity is lower than that of the open-core type of fluxgate (due to demagnetisation), it has been demonstrated that ring-core design can lead to a very low-noise sensor with better long-term stability. Many theories have been proposed in order to explain the operation of ring-core sensors. Analyzing a ring-core as two half-cores and applying a classical description leads to formulae disagreeing with the experimental results. The introduction of demagnetisation yields better results, but the complexity of such analysis grows as time variations of permeability cannot be neglected [1], so far voltage induced in sense winding we must write:

$E_{\mathrm{i}}=-N A \frac{H(1-D)}{(1+[\mu(t)-1] D)^{2}} \frac{\mathrm{d} \mu(t)}{\mathrm{d} t} \mu_{0}$

where $H$ is the measured field, $D$ is the demagnetisation factor, $N$ is the number of turns of the sensing coil, $A$ is the area of the core and $\mu(t)$ is the relative permeability.

A new view of fluxgate mechanism was introduced by Narod and Russel [2]. Instead of flux modulation by the driving current they consider equivalent modulation of sense winding inductance. In quantitative analysis they suppose rectangular excitation current waveform, so that $L(t)$ can acquire only two values. We consider their approach to be sophisticated but restricted to very low values of $H$, for which $L(t)$ can be taken as constant. But the question under what circumstances was it possible to saturate one segment of a ring-core while another part remained unsaturated is as yet unsolved.

Our analysis brings a new attitude to this problem. It is a semi-quantitative description based on a simple approximation of a dynamic loop and derivation of the transfer function similar to that made by Gordon [3]. The main asset of our description is that we take into account the interaction between two segments of a ring core. Here two different magnetisation loops in a ring-core fluxgate are considered:

(1) the "inner" loop, for the toroidal excitation field $H_{\text {exc }}$ characterised here by maximum permeability $\mu$;
(2) the "outer" loop for the homogeneous measured field $H$ which has maximum permeability $\mu^{\prime}$

$\mu^{\prime}=\frac{\mu}{1+(\mu-1) D}$.

Figure 1 indicates the derivation of the transfer function for a given $H$. (Piecewise linear approximation of hysteresis loop.) Let us consider the excitation field growing from zero. For $H_{\mathrm{exc}}=H_{1}=H_{\text {sat }}-H$, half-core 1 falls into saturation, while half-core 2 is unsaturated. At that moment the ringcore is magnetically "broken", the magnetic resistance of sensor seen from the excitation winding rapidly increases, permeability changes from $\mu$ to $\mu^{\prime}$. Both half-cores are saturated for $H_{\text {exc }}=H_{2}$. We derived the shape of the transfer function for different values of $H$.

A series of experiments on several types of ring-core sensors was performed in order to verify the theory. Waveforms of flux in various parts of the sensor core were obtained by integrating voltage induced in the measuring windings. Here results for sensor AT-1 are presented. This is a fluxgate sensor with an oval-shaped closed core (an amorphous $\mathrm{Co}_{75} \mathrm{Fe}_{5} \mathrm{~B}_{20}$ material produced by the Physical Institute, Dresden, GRD) with 20 turns of strip $30 \mu \mathrm{m}$ thick and $2 \mathrm{~mm}$ wide. It is easy to make the sensor even in an oval shape which was selected for its higher sensitivity and ease of measurement because there is no need for high-temperature annealing. It has, however, higher noise level and poorer long-term stability than the classical ring-core sensor made from crystalline materials.

Static inside loops $\Phi_{1}$ vs. $H_{\text {exc }}$ for different $H$ may be seen in Fig. 2. Permeability falling when the half-core 2 is saturated can easily be seen. The last curve for $H=900 \mathrm{~A} / \mathrm{m}$ shows that when $H$ is sufficiently high to saturate the whole sensor even for $H_{\mathrm{exc}}=0$, permeability is $\mu^{\prime}$ only during the whole working cycle.

A dynamic hysteresis loop $\Phi$ vs. $H_{\text {exc }}$ for excitation frequency of $1 \mathrm{kHz}$ is shown in Fig. 3. From the dynamic transfer function sense coil flux vs. $H_{\text {exc }}$ one can see [Fig. 4(a, b)] that the slope is much lower than that of the hysteresis loop, in correspondence with the derivation (Fig. 1). The distance between two peaks is $2 H_{\mathrm{c}}$ for lower fields and grows for $H>H_{\text {sat }}$. Fig. 5(a, b) shows corresponding waveforms in a time domain.

The relation between $H$ and the height of the transfer function (i.e. the change in sense-winding flux during one cycle of excitation current) is linear over a wide range. For understanding this mechanism it is useful to look on fluxgate as the instrument which gates sense-winding flux $\Phi$ by an excitation current. In the case of a one-core sensor, the minimum and maximum flux is determined mainly by $H_{\text {exc }}$. Due to symmetry, these high-level half-core fluxes $\Phi_{1}, \Phi_{2}$ are 

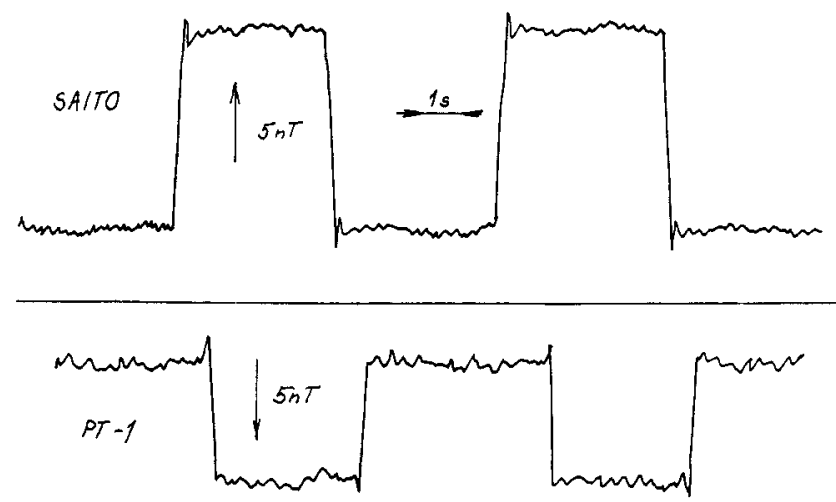

Fig. 8. Noise measured in MAVACS. (a) Sensor Saito, (b) sensor PT-1.

ferromagnetic or active shielding. The former one was convenient to use but it had a slow drift of the field about $1 \mathrm{nT} / \mathrm{min}$ in case of our double permalloy cylindrical shielding due to the relaxation effects. For the latter one the Earth's field compensating system MAVACS (developed by Geofyzika Brno), based on a rotation sensor. It is placed in a magnetically silent laboratory in Průhonice. Long-term zero stability is $0.1 \mathrm{nT}$, noise $30 \mathrm{pT} p-p$. The example of the results is shown in Fig. 8. Measured sensors are:

(a) PT-1 developed in our department - core material Py $79,50 \mu \mathrm{m}$ thick, $6 \mathrm{~mm}$ width, 6 turns;

(b) the sensor developed by T. Saito described in Ref. [4].

\section{Conclusions}

The dynamic transfer functions can be useful not only for understanding the processes in the sensor core, but also in developing new sensors and adjusting sensors and their electronics. We are currently developing a PT-1 based magnetometer with autocalibration abilities and we are trying to increase the stability of the sensor. It would be possible and desirable to test also some other fluxgate developed in another laboratory in MAVACS system to compare performance.

\section{References}

1. Burger, J., IEEE Trans. Magn. 8, 791 (1972).

2. Narod, B. and Russel, D., IEEE Trans. Magn. 20, 592 (1984).

3. Gordon, D., Lundsten, R. and Chiarodo, R., IEEE Trans. Magn. 1, 330 (1965).

4. Saito, T., J. Geomag. Geoelectr. 32, 649 (1980). 